\title{
INTERNET SERVICE PROVIDER-MOBILE BORDER GATEWAY ROUTE OPTIMIZATION TECHNIQUE IN MOBILE IP BASED ON DIFFERENT ISPS STRUCTURES (ISP-MBG)
}

\author{
Sherif Kamel Hussein \\ Department of Communications and Electronics \\ October University for Modern Sciences and Arts \\ Giza - Egypt
}

\begin{abstract}
Due to the proliferation in the number of users that are accessing the internet and due to the increase in the number of the electronic devices that support mobility like mobiles, laptops and many others that definitely lead to the need of a protocol that supports a mobility. Mobile Internet Protocol is a recommended Internet protocol designed to support the mobility of a user (host). This protocol provides a continuous connectivity for any mobile host. In the traditional Mobile IP all packets forwarded to the Mobile host from the correspondent node will be forwarded via the Home Agent (HA) and that leads to the triangle routing problem.

(ISP MBG) technique is used as a route optimization technique for solving the triangle routing problem in conventional Mobile IPv4. This technique has been implemented on .net platform .The study of this technique was discussed before using 2 similar Internet Service Providers and the simulation results provided a better performance compared with the Conventional Mobile IP Technique. In this paper the simulator will be used to study the performance of the (ISP MBG) technique using two different Internet Service Providers ( ISPS) structures separated by a single Mobile Border Gateway ( MBG).Simulation results shows also a better performance compared with the conventional Mobile IP technique .
\end{abstract}

\section{KEYWORDS}

Internet Service Provider, Point of Presence, Mobile IP Border Gateway, PoPs Virtual Mobile IP Network.

\section{INTRODUCTION}

Mobile IP is an open standard protocol defined by the Internet Engineering Task Force (IETF) RFC 2002. That allows users to keep the same IP address, stay connected, and maintain ongoing applications while roaming or travelling between networks. The operation of Mobile IP protocol is based on three main functions, Agent Discovery, Registration and Tunneling [1].

The main problem of Mobile IP is the triangle routing problem in which all the traffics sent by the Correspondent node $(\mathrm{CN})$ to Mobile Node $(\mathrm{MN})$ will be intercepted by the Home Agent (HA) which is a main router serving the home network for the Mobile Node .Home Agent will tunnel the transmitted packets to the Foreign Agent (FA) which is a router serving the foreign

DOI : $10.5121 /$ ijcnc.2014.6406 
network where the mobile node is located .The Foreign Agent then de- tunnels the received packets and forward the packets finally to the Care of Address (CoA) of the Mobile Node on the foreign Network [2].

The route taken by the transmitted packets from the Mobile Node to the Correspondent Node is Triangular in nature which definitely leads to an increase in the delay time and also decrease in network throughput. Internet Service Provider Mobile Border Gate Way ( ISP MBG) is a route optimization technique based on using multiple Internet Service Providers (ISPs) separated by a multiple Mobile Border Gateways (MBGs). This technique has been studied and discussed before based on using two similar Internet Service Providers structures and the simulation results showed a better performance compared with the conventional Mobile IP technique. In This paper the technique will be studied based on using two different Internet Service Providers Structures[3-4].

The paper is divided into five sections. The second section: introduces the route optimization technique Internet Service Providers Mobile Border Gateway (ISP MBG) for two similar ISP structures . The third section: presents the implementation of ISP MBG technique based on two different ISPSs Structures. Section 4: introduces the evaluation and the simulation results for the proposed ISP MBG technique compared with conventional Mobile IP technique. Conclusion and future work are presented in section 5.

\section{INTERNET SERVICE PROVIDER MOBILE BORDER GATEWAY TECHNIQUE [5]}

The design is composed of a number of $\mathrm{N}$ Internet Service Providers $\mathrm{ISP}_{1}, \mathrm{ISP}_{2}, \ldots \ldots \ldots, \mathrm{ISP}_{\mathrm{N}}$, each covers a definite and different geographical place. They are separated by an L Mobile IP Border Gateways (MBGs) .Each ISP is serving S equal Areas. Each Area is divided into M Zones and each Zone is served by $\mathrm{K}$ number of Points of Presence nodes (PoPs).Each PoP is serving $\mathrm{X}$ number of nodes with a range $\mathrm{W}$ of Addresses. The nodes are classified as either Local Nodes or Foreign Nodes based on whether they are located in the same Agent or in different Agents. Each Area is served by an Agent which is considered as a Home Agent for the local nodes inside the Area or as a Foreign Agent for the nodes located outside the Area .For each Internet Service Provider there are K PoPs Virtual Networks (PVNs) which handle the state information for all Mobile Nodes and Correspondent Nodes. Figure 1 show the structure for a given ISP . Figure 2 show an example of PoPs Virtual Networks (PVNs) for a given ISP.

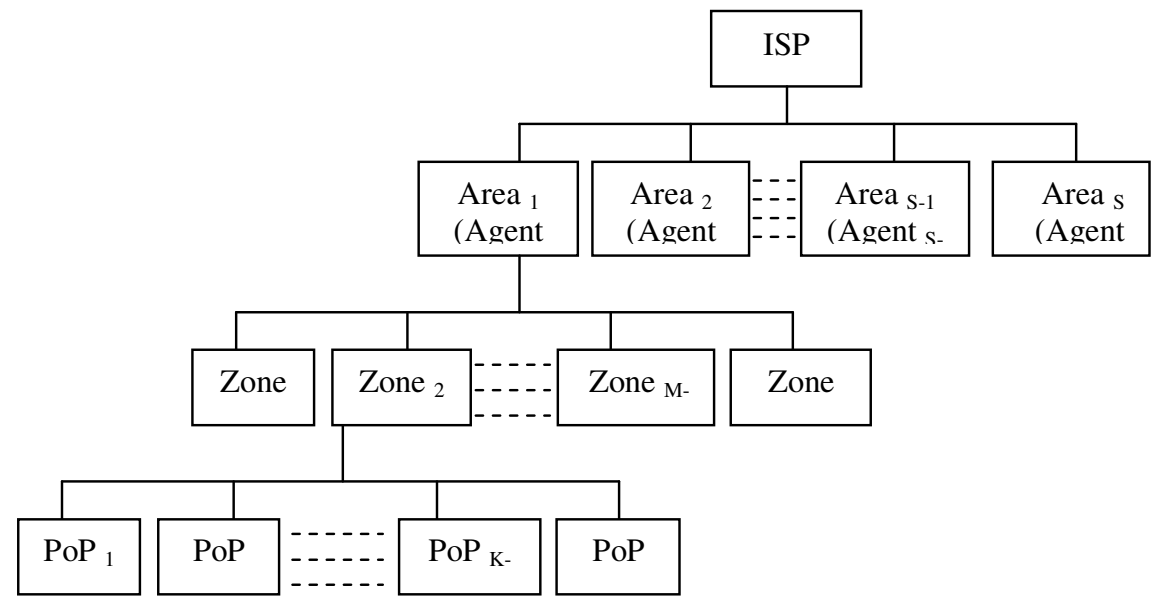

Figure 1. Structure For a Given ISP 


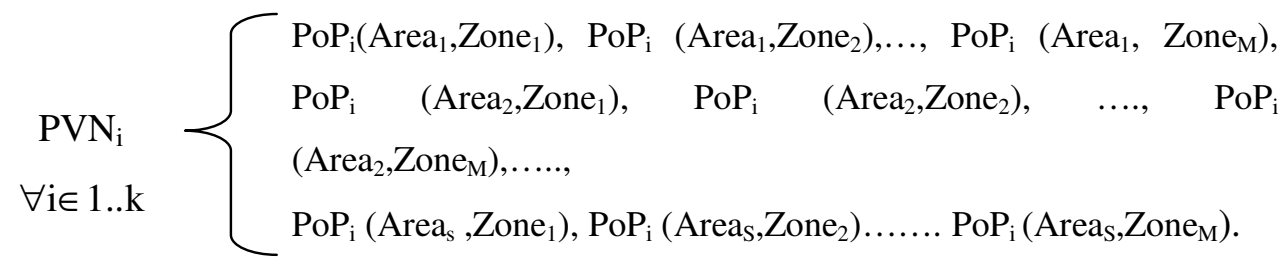

Figure 2. PoPs Vitual Network for a Given ISP

ISP MBG technique is supporting both the tunneling and routing techniques.MBG will either maintain the binding information (Home address, Care-of-Address) or only the home information based on whether the ISP MBG technique is using tunneling or routing technique in forwarding the packets between the Correspondent Nodes and the Mobile Nodes [5-6].

\subsection{ISP MBG Technique Operation Sequence}

When a connection is to be started between two nodes the Correspondent Node and Mobile Node, the execution of the ISP MBG technique will be started by checking the location of both nodes.If both nodes are located in the same Internet Service Provider (ISP) so the Correspondent Node will start asking for an information about the Mobile Node by connecting first to its nearest home PoP. That PoP will search its neighboring PoPs through the PoP Vitual networks. One of these PoPs should have an information about the Mobile Node and the connection will be started. In case if both MN and CN are in different ISPs, the same sequence will be taken but the only difference is that the PoP that is connected to the MN through the PoP virtual network (PVN) will connect to Mobile Border Gateway (MBG) which maintains the original home information for the destined MN.MBG will then connect to the nearest PoP in the destination ISP which is connected to a new virtual Network of the Mobile Node and then the connection will be started directly between the Correspondent Node (CN) and the Mobile Node (MN).Figure 3 shows the operation sequence for ISP MBG technique in both cases . In case 1 both $\mathrm{MN}$ and $\mathrm{CN}$ are in the same Agent but in case 2 both $\mathrm{MN}$ and $\mathrm{CN}$ are in different Agents [5-6].

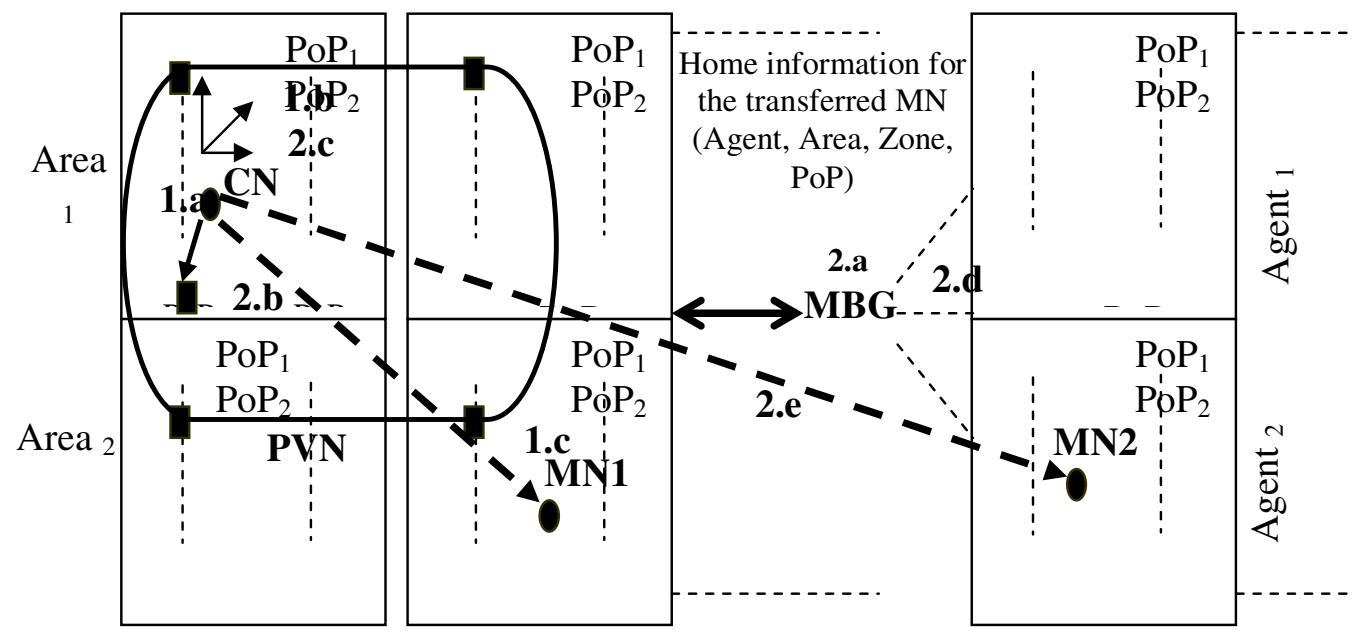

Figure 3. Operation Sequence for the Proposed Technique 


\subsection{ISP MBG System Parameters}

The key point to establish any System is to define the system parameters .System Parameters include the simulation parameters, data structures, and the connection parameters. Simulation parameters are related to the system construction and defined by the number of ISPs, MBGs, Areas, Zones, PoPs, and the in service or in waiting nodes covered by each PoP .The main data structures used in the simulation are Nodes, POPs, Areas, Agents, and Mobile IP Border Gateway (MBG). All the data structures are implemented in C sharp on a microsoft.net platform [5-6].Finally the connection parameters are the parameters required for the design and the implementation of the ISP MBG algorithm and are classified as follows:

1. The distance in $\mathrm{km}$ equivalent to the distance of 1 pixel.

2. Link speed for PoP connection

3. Link speed for Agents connection

4. PoP nodes count to serve.

5. PoP nodes count to wait

6. Agent nodes count to serve

7. Agent nodes count to wait

\section{IMPLEMENTATION OF ISP MBG TECHNIQUE BASED DIFFERENT ISPS STRUCTURES.}

The Implementation of the ISP MBG technique is based on two different ISPs Structures. The first ISP is serving only one Area with 2 Zones and 4 PoPs per Zone. The second ISP is serving 2 similar Areas with 3 Zones each, and 3 PoPs for every Zone. Each PoP is actually serving 100 nodes with 150 addresses as shown in figure 4 . The addresses are classified as 100 addresses available for the nodes in service and the remaining 50 Addresses for the nodes that are in the waiting state.For the nodes in service we can split the addresses into 75 addresses for local generating nodes and 25 addresses for external nodes. In a similar way the waiting nodes addresses are divided equally between the local and the external generating nodes. The number of nodes used in the simulation is about 2000 Nodes. Figure 5 shows the design architecture of the proposed ISP MBG technique based different IPSs structures.

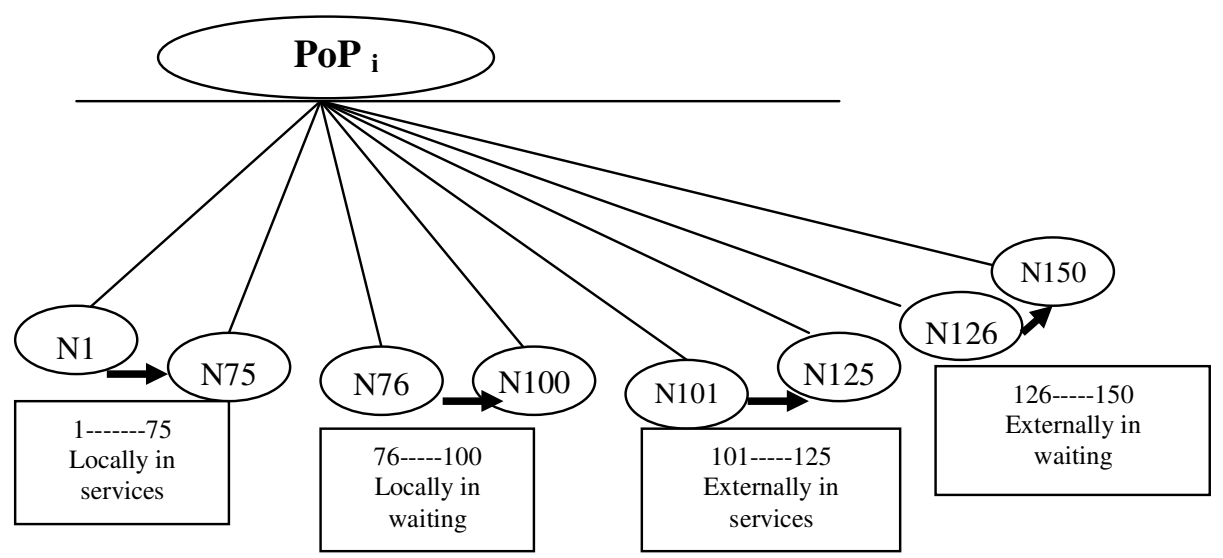

Figure 4. Nodes and Address Classifications for $\mathrm{PoP}_{\mathrm{i}}$ 
The total number of PoPs can be calculated by the following equation:

$$
\mathrm{PN}=\sum \mathrm{Pik} \mathrm{X} \mathrm{Niz}
$$

Where;

$\mathrm{PN}$ is the total number of PoPs

Pik is the number of PoPs within the zone in Internet Service Provider k

$\mathrm{Ni}$ is the total number of Zones in Internet Service Provider $\mathrm{i}$

Also the Applicable total number of generating nodes can be calculated from the given equation.

$$
\mathrm{NT}=\mathrm{PN} \times \mathrm{NC}
$$

Where;

NT is the total number of generating nodes

NC is the total number of generating nodes in each connection.

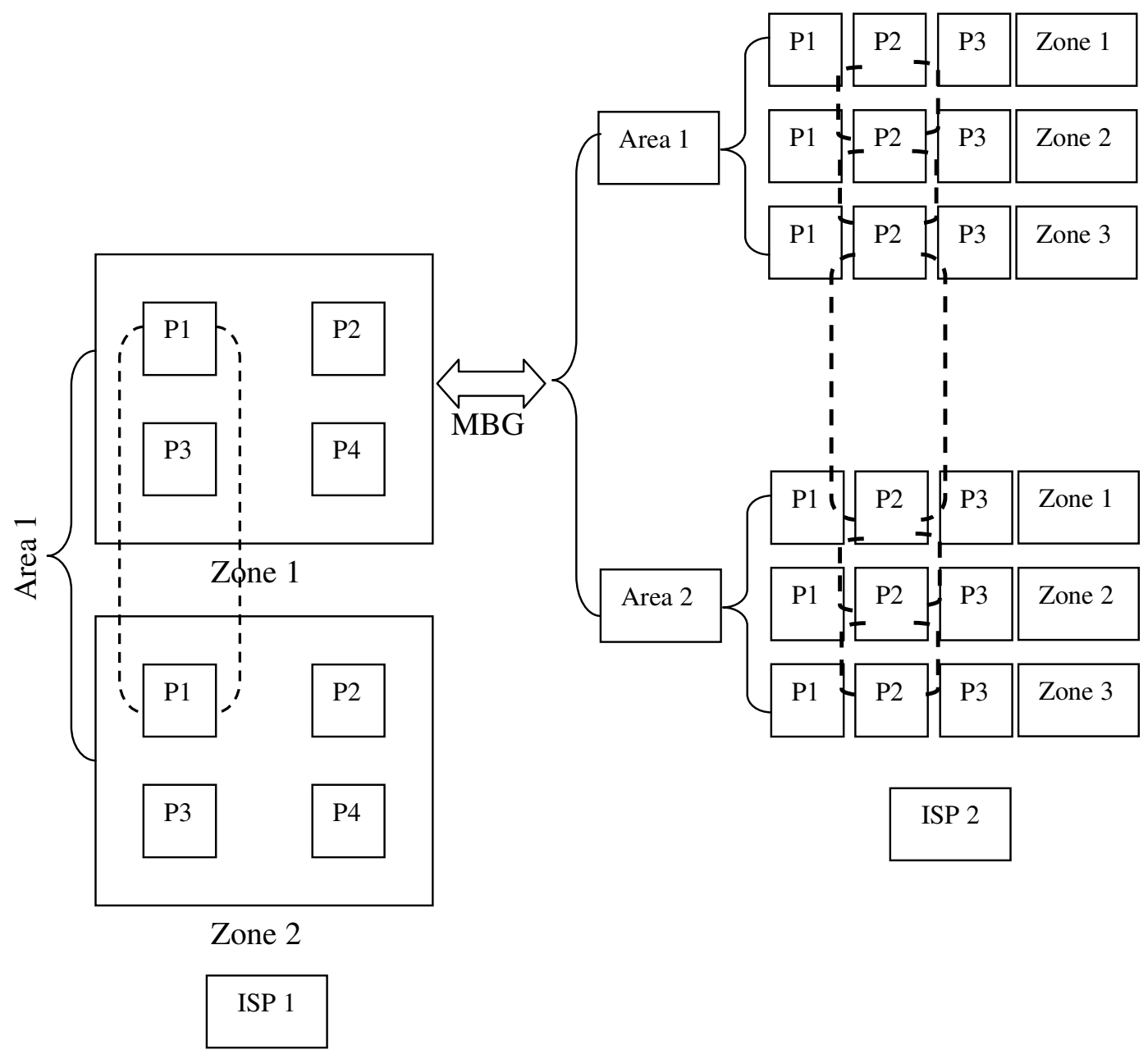

Figure 5. System Construction 
International Journal of Computer Networks \& Communications (IJCNC) Vol.6, No.4, July 2014

\section{EVALUATION OF THE ISP MBG TECHNIQUE}

To evaluate the performance of the proposed ISP MBG technique, the following five measuring criteria are required: Link Distance, Transmission Time, Blocking, Buffering and Security.Link distance will be calculated based on the distance between two connecting nodes and it is calculated in kilometer equivalent to the number of pixel $(1$ pixel $=0.2 \mathrm{Km})$. The Link distance is calculated using the following equation:

$$
\begin{gathered}
\mathbf{D}_{\mathbf{t}}\left(\mathbf{P}_{\mathbf{i}}, \mathbf{P}_{\mathbf{z}}\right)=\sum D\left(P_{m}, P_{m+1}\right) \\
\forall m \in i \ldots z-1
\end{gathered}
$$

Where; P: Transmission point (PoP or MBG)
D: Euclidian distance
$\mathrm{D}_{\mathrm{t}}$ : Total link distance between two transmission points

The transmission time will be calculated based on the location of both nodes, the Mobile Node $(\mathrm{MN})$ and the Correspondent Node $(\mathrm{CN})$ and whether both are located in the same area, same Internet Service Provider or either one in different areas or different Internet Service Providers. the transmission time will be calculated using the following equation:

$$
\mathrm{T}\left(\mathrm{P}_{\mathrm{i}}, \mathrm{P}_{\mathrm{Z}}\right)=\sum_{\forall m \in i \ldots . . Z-1} \frac{D\left(P_{m}, P_{m+1}\right)}{L_{m}}
$$

Where; $\quad$ P: Transmission point (PoP or MBG)

$\mathrm{T}\left(\mathrm{P}_{\mathrm{i}}, \mathrm{P}_{\mathrm{z}}\right)$ :Total transmission time between two points $\mathrm{P}_{\mathrm{i}}$ and $\mathrm{P}_{\mathrm{z}}$

L: Link speed

D: Euclidian distance

Blocking will be calculated as the number of blocked connections and that will define the network throughput taking into consideration that each connection has 2 nodes. Buffering is considered as one of the network resources that must be optimally used. In conventional Mobile IP buffering is based mainly on the storage buffers of the Agents whatever Home or foreign Agent but in the proposed technique mainly is based on the PoPs storage Buffers and the PoPs virtual Networks. Security is an important performance parameter to measure how much the data and the information are secured while travelling from one network to another. Further study for the performance of the proposed technique will be used to measure the evaluation for both the buffering and the security parameters [7-8].

\subsection{Simulation Results for the case of 2 different ISPs structures}

For the case where two different ISPs are used, the first ISP consists of one Area of two Zones and four PoPs per Zone, and the second ISP consists of two Areas, each with three Zones and three PoPs per Zones, the algorithm is applied for a range of 2000 Nodes The comparison graphs will be detailed in the following subsections . 


\subsubsection{Simulation Results for Link distance}

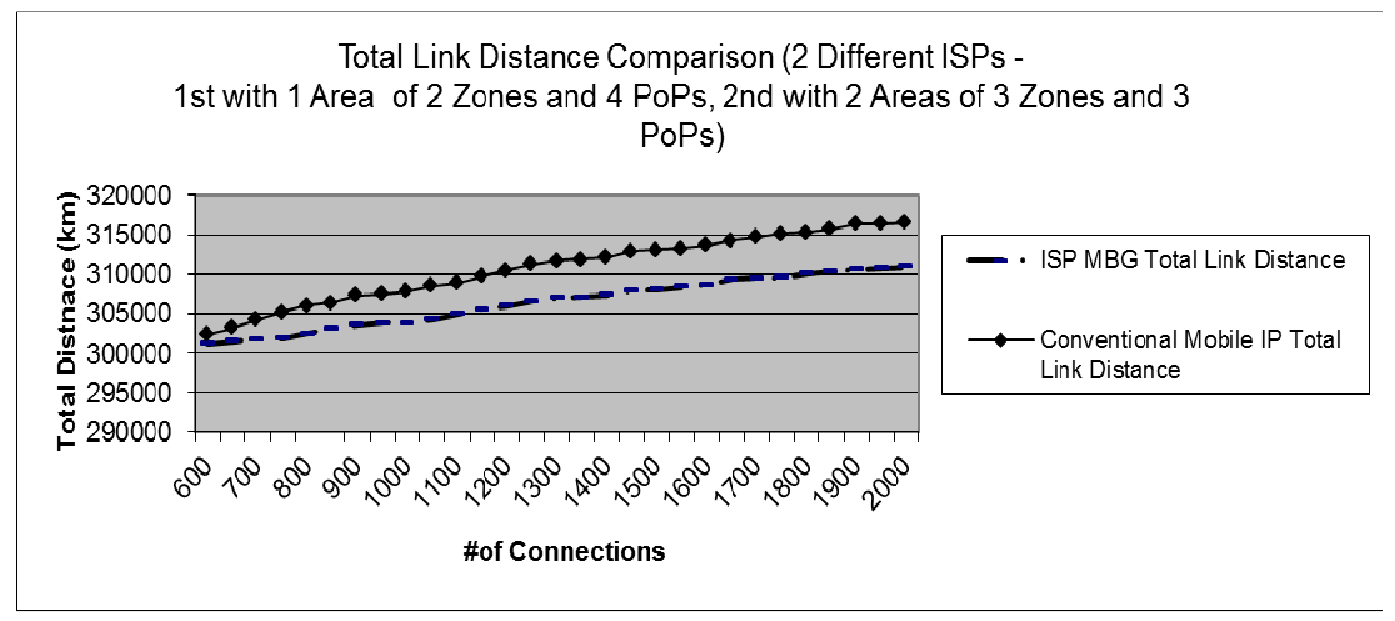

(a) Total Link Distance for the Conventional and the ISP MBG Techniques

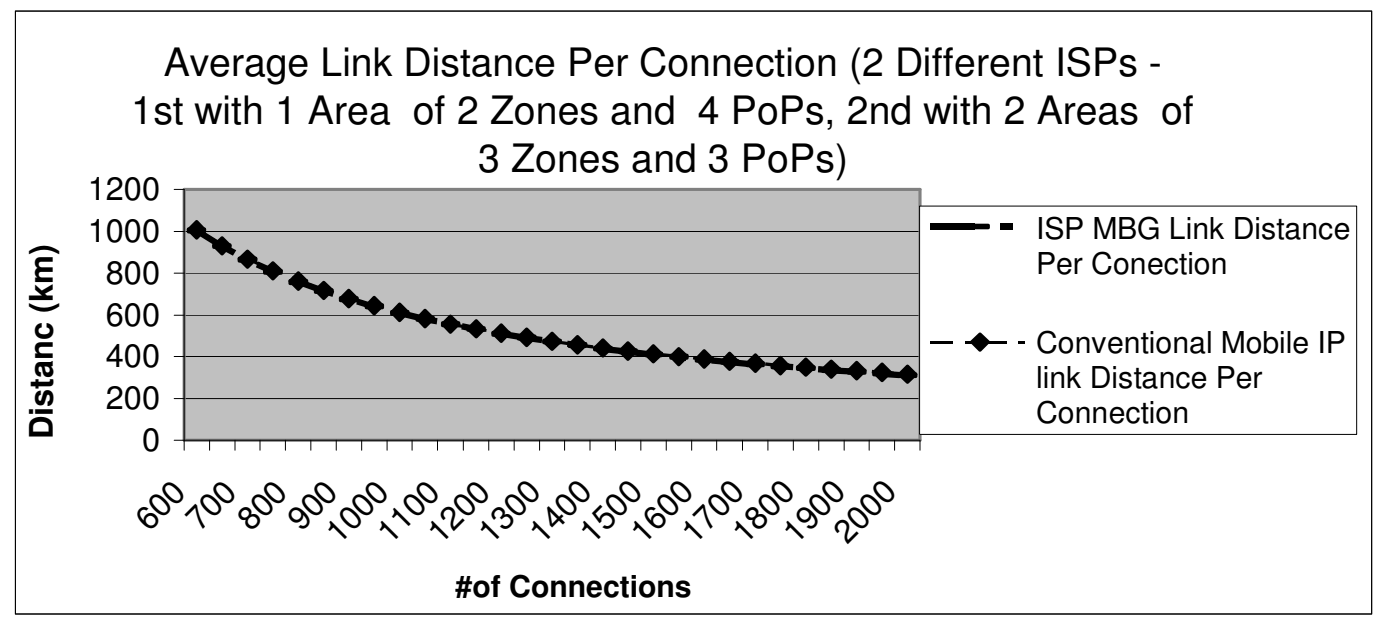

(b) Average Link Distance for the Conventional and the ISP MBG Techniques

Figure 6. Link Distance Comparison

Form figures (6.a) and (6.b) we can see that, when different ISPs with different configurations are connected to each other, the performance of the new technique still outperforms the conventional techniques. 


\subsubsection{Simulation Results for Transmission Time}

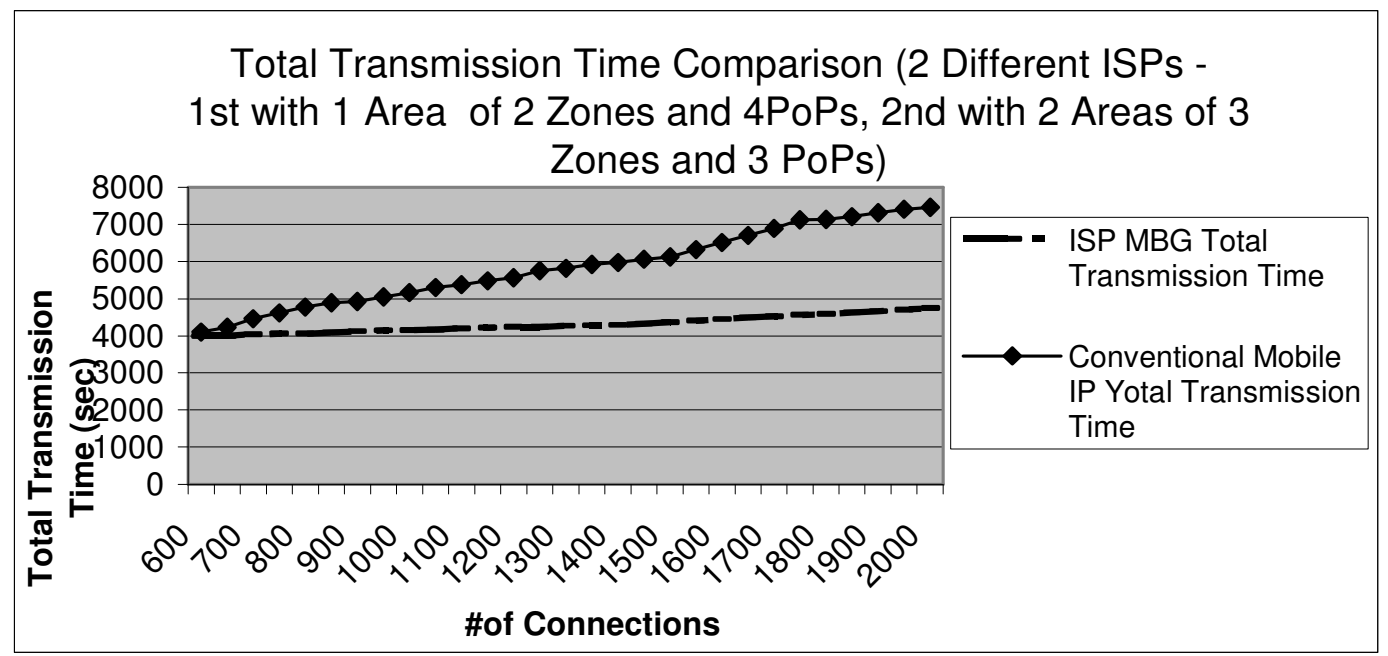

(a) Total Transmission Time for the Conventional and the ISP MBG Techniques

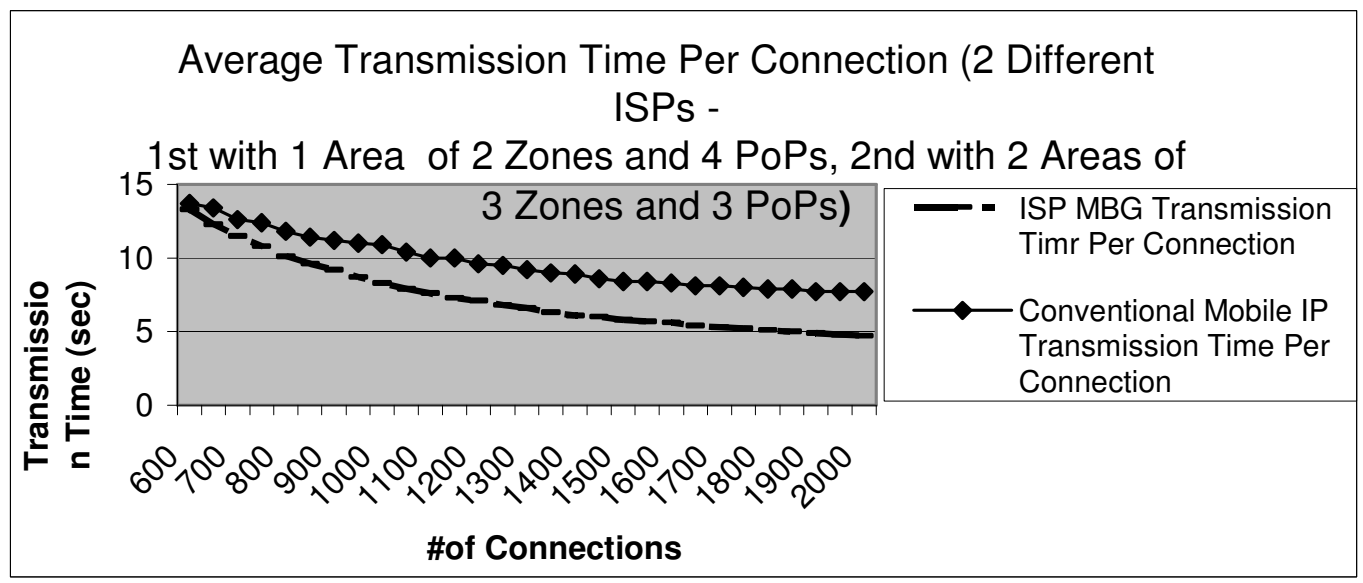

(b) Average Transmission Time for the Conventional and the ISP MBG Techniques

Figure 7. Transmission Time Comparison

Figures (7.a) and (7.b) show that the same argument applies when comparing the transmission time performance and the results for the link distance simulation, the new technique outperforms the conventional technique in the transmission time . 


\subsubsection{Simulation Results for Throughput}

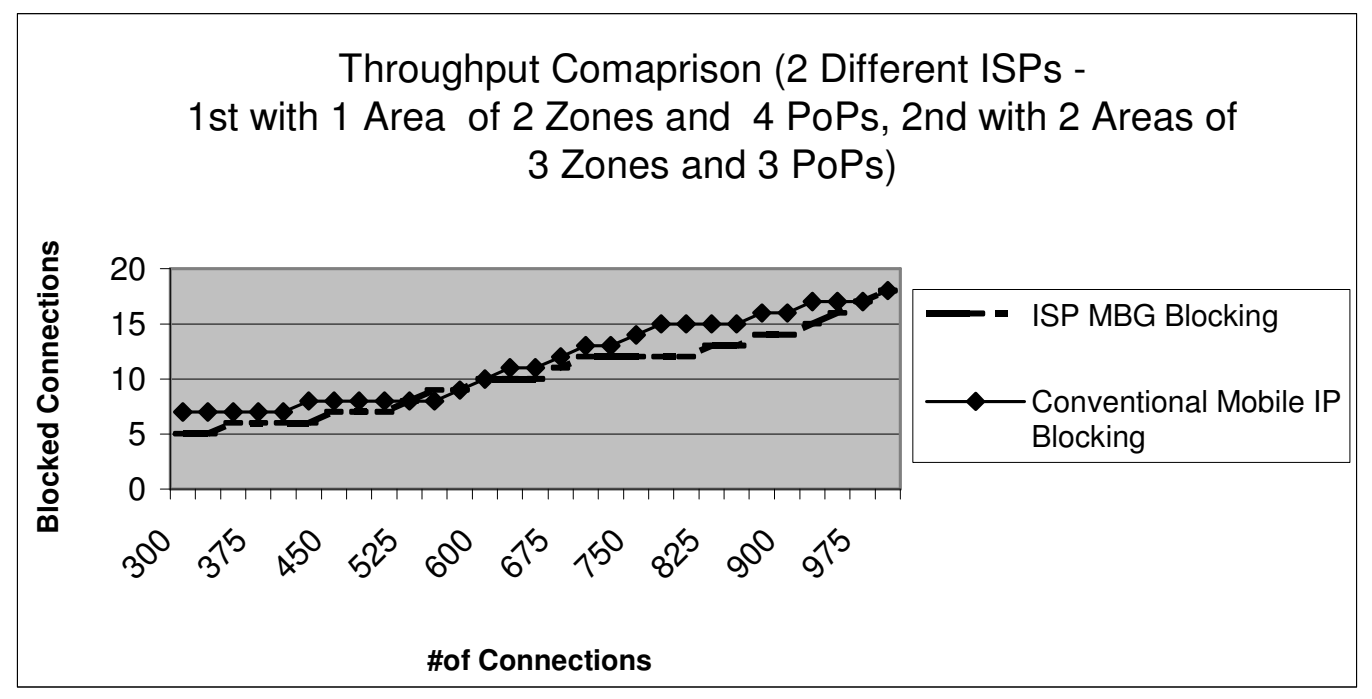

Figure 8. Throughput Comparison

Figure 8 shows the throughput performance of the new techniques is still better than the conventional techniques, similar to the case of same ISPs.

\subsubsection{Buffering}

Concerning Buffering; it has been found that the ISP MBG technique provides better buffering than the Conventional Mobile IP technique. Using PVN is facilitating the process of accessing the home information for any mobile nodes roaming between networks . Because of PVN provides a virtual network among PoPs, the home information of Mobile Node could be accessed without any redundancy of having multiple copies at each PoP .Comparatively in the conventional Mobile IP technique the Home Agent Buffer should maintain the home information for each node travelled to a foreign network and that definitely lead to the need of high buffering size. On the other side it has been found that the cost for the Agent's buffers is higher than that of the PoP's buffers [9].Further study will be conducted in the future to elaborate in more details the comparison between buffering in both the conventional and the proposed ISP MBG techniques.

\subsubsection{Security}

The Security design has a great concern in Mobile IP. In ISP MBG technique Mobile IP Border Gateway (MBG) is used to keep the information (Home address or binding information) for all Mobile Nodes roaming from one network to another so there will be no need for any Correspondent Node $(\mathrm{CN})$ to maintain any private information for the new IP network where the Mobile Node is visiting . Comparatively, the conventional Mobile IP technique needs high level of security to prevent the malicious users from intercepting the connection between the $\mathrm{MN}$ and the $\mathrm{CN}$ which maintains the binding information (Ha, CoA). Further study will be conducted in the future to Study the comparison between Security level in both the conventional and the proposed ISP MBG techniques . 
International Journal of Computer Networks \& Communications (IJCNC) Vol.6, No.4, July 2014

\subsection{Performance Comparison Between The Conventional Mobile IP Technique And the Proposed ISP MBG Technique Based Different IP Structures}

Table1 summarize the performance comparison between the conventional Mobile IP technique and the proposed ISP MBG techniques for different ISP structures respectively.

Table1. Comparative Parameters for the Conventional and the Proposed ISP MBG Techniques

[Different ISPs structures]

\begin{tabular}{|l|l|l|}
\hline \multicolumn{1}{|c|}{ Technique } & \multicolumn{1}{c|}{$\begin{array}{c}\text { ISP MBG } \\
\text { Technique }\end{array}$} & \multicolumn{1}{c|}{$\begin{array}{c}\text { Conventional Mobile IP } \\
\text { Technique }\end{array}$} \\
\hline Link Distance & Shorter Link distance & Longer link distance \\
\hline Transmission Time & Lower transmission time & Higher transmission time \\
\hline Blocking & lower rate of blocking & Higher rate of blocking \\
\hline Buffering & $\begin{array}{l}\text { - Less buffering storages } \\
\text { - Lower cost }\end{array}$ & $\begin{array}{l}\text { - More buffering storages } \\
\text { - Higher cost }\end{array}$ \\
\hline Security & Higher level of security & Lower level of security \\
\hline
\end{tabular}

\section{CONCLUSION AND FUTURE WORK}

In this paper, a further study on the ISP MBG Route Optimization Technique has been introduced to check the performance evaluation of the technique when two different ISPs structures are used. The design and the sequence of operation for ISP MBG technique are reviewed in this paper. The implementation of the case study for two different ISPs structures is also detailed in this paper .

For different ISPs structures, the simulated network design of our case study is based on using two different Internet Service Providers structure separated by one Mobile IP border gateway. One ISP is composed of one Area, 2 Zones and 4 PoPs serving each Zone. Another ISP is composed of 2 Areas, each has 3 Zones and 3 PoPs serving each Zone. Each PoP is serving 100 nodes with a range of 150 addresses. The simulation results for the Link Distance, Transmission Time ,Blocking, Buffering, and Security show that the proposed (ISP MBG) technique still outperforms the conventional Mobile IP technique.

This work can be extended to include investigation of using multiple ISPs and multiple MBGs. In that case the MBG is not only restricted to hold the home information for the nodes crossing their local ISP, or to guide in establishing the route to the MN located in an external ISP, but rather the investigated subjects in that areas are continuing enhance the functionalities of MBG to do more advanced tasks. Such tasks include, tunneling, conditional Processes for the route optimization, and others.Moreover, using multiple MBGs will distribute the processing load among the MBGs.

\section{REFERENCE}

1. Abbas Jamalipour, ( 2003) “The Wireless Mobile Internet”, John Wiley \& Sons Ltd., England.

2. Philip J. and Nesser II, (2001) "Survey of IPV4 Addresses in Currently Deployed IETF standards", Internet-drafts, draft-ietf-ngtrans-ipv4 survey-01.text, work on progress. 
International Journal of Computer Networks \& Communications (IJCNC) Vol.6, No.4, July 2014

3. Sherif kamel Hussein, Khaled Mohamed Almustafa, "Triangle Routing Problem in Mobile IP", Journal of Communication and Computer, David Publishing ,JCC-E20140114-3 USA,2014.

4. Ramesh Bharti, Vishnu Kumar Sharma, Navneet, Aman Gupta4, (2013) "A P2P Based Route Optimization Architecture for Mobile IP Based on Simulation Work", International Journal of Emerging Technology and Advanced Engineering Website: www.ijetae.com (ISSN 2250-2459, ISO 9001:2008 Certified Journal, Volume 3, Issue 2.

5. Sherif Kamel Hussein, (2012) "Extended Study on ISP MBG Route Optimization Technique in Conventional Mobile IP 4 ", IOSR Journal of Computer Engineering (IOSRJCE) ISSN: 2278-0661 Volume 3, Issue 6 (Sep-Oct. 2012), PP 20-35 ww.iosrjournals.org.

6. Rajesh Singh Thakur, Babita Kubde, (2013)" Implementation of Route Optimization Mobile IP to Analyze the TCP Performance during Handoff", International Journal of Science and Research (IJSR), India Online ISSN: 2319-7064,Volume 2 Issue 1.

7. Hero Modares, Amirhossein Moravejosharieh, Rosli Salleh, (2013) “ Secure Connection in Mobile IPv6" Life Science Journal.

8. Susanna S Henry, Dr. V. Santhosh Kumar, (2012) "Current status of Mobile Internet Protocol Version 4 and its security Issues “,IJCSI International Journal of Computer Science Issues, Vol. 9, Issue 2, No 2,

9. www.Cisco.com golLogistics

\section{AUTHORS}

Sherif kamel Hussein Hassan Ratib : Graduated from the faculty of engineering in 1989 Communications and Electronics Department ,Helwan University. He received his Diploma MSc,and Doctorate in Computer Science ,Major Information Technology and Networking. He has been working in many private and governmental universities inside and outside Egypt for almost 13 years .He shared in the development of many industrial courses .His research interest is GSM Based Control and Macro mobility based on Mobile IP .

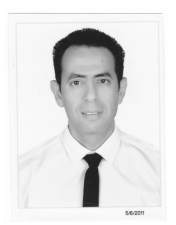

